\title{
Rupture ou continuité ?
}

\section{Rupture or continuity?}

\section{Mitanchez}

(C) Springer-Verlag France 2012

Lorsqu'il y un an le Pr Lequien me contactait pour me demander de lui succéder à la rédaction de la revue, j'avoue avoir été surprise de ce choix, puis très touchée devant l'argumentation de mon interlocuteur! Après quelques jours de réflexion, j'ai finalement accepté cette mission.

La revue est jeune, mais elle se porte bien Ce n'est pas un embryon, mais bien un foetus en pleine croissance comme le montre son l'évolution sur deux années. Cinquante et un articles ont été publiés en 2011 , soit presque $25 \%$ de plus qu'en 2010. De même, le nombre de téléchargements d'article a augmenté d'un tiers en 2011. Cela démontre l'intérêt grandissant pour cette revue, tant pour les auteurs que pour les lecteurs. Cela est aussi le résultat du dynamisme et de l'implication du comité de rédaction et du comité scientifique, animés pendant trois ans par le Pr Lequien avec l'énergie et l'humour qu'on lui connaît.

Devant un tel constat, il ne peut être question de rupture même si je reconnais avoir quelques différences avec mon prédécesseur, mais de continuité. Il s'agit en effet d'assurer dans la continuité le développement, la diffusion et la reconnaissance institutionnelle de la revue.

Il est probable que ma disponibilité ne sera pas égale à celle du Pr Lequien, mais cela ne doit pas nuire à mon engagement. C'est pour cela que j'ai souhaité mettre en place une rédaction bicéphale. J'ai à mon tour sollicité le Pr Bruno
Carbonne, obstétricien, avec qui j'ai la chance de collaborer depuis quelques mois à l'hôpital Trousseau. Il a accepté le poste de rédacteur adjoint. Il me semblait que cette association était légitime dans le contexte et qu'elle permettra d'améliorer la contribution obstétricale à la revue. Les articles obstétricaux sont les moins nombreux mais paradoxalement, parmi les dix articles les plus téléchargés, une majorité est consacrée à l'obstétrique. La revue de médecine périnatale peut donc être pour les plus jeunes un moyen de se faire entendre et de se faire connaître.

Le comité de rédaction a été élargi pour renforcer la représentation de certaines spécialités. Je remercie Agnès Rigouzzo, anesthésiste et Annie Serfaty, médecin de santé publique qui ont accepté de venir travailler à nos côtés. Elles ont déjà eu l'occasion d'apprécier la richesse des échanges et la convialité qui règnent au sein des comités.

Enfin, n'oublions pas que la Revue de médecine périnatale est l'organe de la SFMP. Le travail se fera donc en lien avec la société dont beaucoup d'acteurs participent déjà à la vie de la revue.

Je remercie donc le Pr Lequien pour sa confiance et je m'efforcerai d'être à la hauteur de ses espérances. Je remercie aussi le conseil d'administration de la SFMP d'avoir validé ce choix et de me laisser aux manettes de ce bel outil plein d'avenir.

D. Mitanchez $(\bowtie)$

Service de néonatologie, hôpital Armand Trousseau, 26, avenue du Dr Arnold Netter, F-75012 Paris

e-mail : delphine.mitanchez@trs.aphp.fr 